\title{
Towards Autonomic Network Management for Mobile IPv4 Based Wireless Networks*
}

\author{
Dong-Hee Kwon, Woo-Jae Kim, Young-Joo Suh, and James W. Hong \\ Departments of Computer Science and Engineering \\ Pohang University of Science and Technology (POSTECH) \\ \{ddal, hades15, yjsuh, jwkhong\}@postech.ac.kr
}

\begin{abstract}
The rapid progress of wireless communication technologies has opened a possibility to offer various types of communications to users irrespective of their locations. The all-IP based wireless networks have been proposed and the Mobile IP protocol is considered as one of prominent candidate frameworks to support a seamless mobility of users. However, to our best knowledge, there are few research efforts to design and develop a network management system targeted for Mobile IP based wireless networks. In this paper, we introduce the concept of autonomic wireless network management which utilizes SNMP agents to manage more intelligently through the selfmanagement functionality. We present a design of autonomic wireless network management system (AWNMS) and its prototype implementation. The currently implemented system is fully compliant with Mobile IP MIB and provides management functions such as network topology auto-discovery, mobility tracking function, etc. The implemented system is validated and examined in a Wireless LAN based test-bed network.
\end{abstract}

\section{Introduction}

The rapid progress of wireless data communication technologies ranging from Wireless Local Area Networks (WLANs; e.g., IEEE 802.11[1]) to Wireless Metropolitan Area Networks (WMANs; e.g., IEEE 802.16 [2]) and third-generation cellular systems [3] have opened a possibility to offer various types of communications to users irrespective of their locations. This development tendency of wireless network technologies has accelerated the diversity of wireless network devices and allowed user devices to move freely within large geographical areas and changed the form of handover management. The handover management in wireless networks such as cellular networks is carried out by technology specific mechanisms since only the intra-technology handovers are involved given the same type of wireless network and single mode terminals. However, the need for integrating different types of wireless networks and the emergence of multi-mode enabled mobile

\footnotetext{
* This research was supported by the MIC (Ministry of Information and Communication), Korea, under the ITRC (Information Technology Research Center) support program supervised by the IITA (Institute of Information Technology Assessment)" (IITA-2005- C1090-0501-0018) and by the Electrical and Computer Engineering Division at POSTECH under the BK21 program of the Ministry of Education, Korea.
} 
terminals have pushed the traditional handover functionality at L2 layer to the generic IP layer that serves the rendezvous point of underlying wireless technologies. Accordingly, the need for migration of technology specific core infrastructures to allIP based networks has been identified and great efforts are placed in this direction. Mobile IPv4 [4] and Mobile IPv6 [5] which have been standardized by Internet Engineering Task Force (IETF) are the results of such efforts.

In general, a network management system provides useful functionalities and information to network administrators for efficiently managing target networks. Simple Network Management Protocol (SNMP) [6] is one of the most widely used protocols for IP based network management. SNMP enables network administrators to manage network performance, find and solve network problems, and plan for network growth. The management functional areas that should be provided by network management systems are fault, accounting, configuration, performance, and security [7]. The Mobile IP based wireless networks also need a network management system to efficiently manage and use networks. However, to our best knowledge, there are few research efforts to design and develop a network management system on mobile wireless networks, typically focusing on the Mobile IPv4 protocol. In this paper, we design and implement a network management system for the Mobile IPv4 based wireless networks.

We first introduce the concept of autonomic wireless network management which utilizes SNMP agents to manage more intelligently through the self-management functionality. The SNMP agents in our autonomic wireless network management system (AWNMS) exchange network information such as network resource status and security information with neighbor agents. In the mobile wireless network, the seamless mobility is important to users for continuous communication during handovers. IETF has standardized new protocols such as the Candidate Access Router Discovery (CARD) [9] for supporting the seamless mobility. However, in the proposed AWNMS, we integrate these functionalities to SNMP agents using the selfmanagement function and do not require such new protocols for seamless mobility.

In this paper, we also present results of the prototype implementation based on our Mobile IPv4 protocol stack. The currently implemented system is fully compliant with Mobile IP MIB [8] and provides management functions such as network topology autodiscovery, mobility tracking function, etc. To support these management functions, our AWNMS uses the integrated information from multiple managed objects for providing advanced functions in the wireless networks. The implemented system is validated and examined in a Wireless LAN based test-bed network.

\section{Related Work}

Mobile IPv4/IPv6 [4, 5] proposed by IETF provides a basic node mobility management scheme in IP networks. When a mobile node (MN) moves from one subnet to another ((1) in Fig. 1), it requests its home agent (HA) to update its binding to receive continuous service ((2) binding update in Fig. 1). In case of Mobile IPv6, configuration of new care-of-address $(\mathrm{CoA})$, which denotes the current location of the $\mathrm{MN}$, is required prior to sending a Binding Update message to HA. A binding maintained by the HA is an association of a MN's home address and its CoA. When 
the HA has the binding for the $\mathrm{MN}$, the HA intercepts any packets destined to the $\mathrm{MN}$, and tunnels them to the MN's CoA ((3) and (4) in Fig. 1). Thus, it is necessary for the $\mathrm{MN}$ to register its current point of attachment to the HA whenever it handovers to another network. Thus, the Mobile IP scheme enables the MN to move freely in the Internet without any disruption of data service.

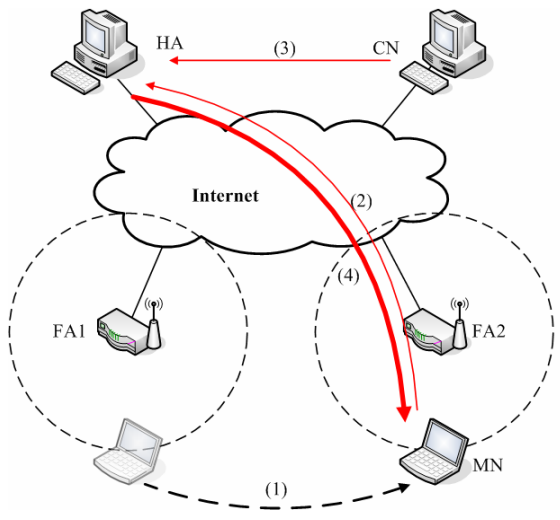

Fig. 1. Mobile IP operation

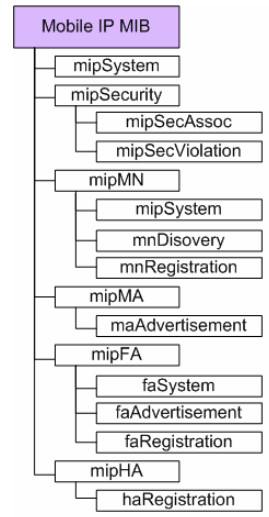

Fig. 2. Mobile IP MIB

To achieve this, three entities (HA, FA, and MN) defined in Mobile IPv4 should interact with each other by exchanging signaling messages to update and synchronize important information such as the state of each entity and the binding information. To endow a network management system to control and monitor the protocol operation of Mobile IPv4, the definitions of Managed Objects for IP Mobility Support using SMIv2 (Mobile IP MIB) has been standardized [8].

Table 1. Relationships between Mobile IP MIB groups and entities

\begin{tabular}{|c|c|c|c|}
\hline & MN & FA & HA \\
\hline mipSystem & O & O & O \\
\hline mipSecurity & O & O & O \\
\hline mipMN & O & & \\
\hline mipMA & & O & O \\
\hline mipFA & & O & \\
\hline mipHA & & & O \\
\hline
\end{tabular}

The Mobile IP MIB, shown in Fig. 2, consists of six main groups which are related to the HA, FA, and MN. The relationships between six groups and three entities are shown in Table 1. First of all, mipSystem and mipSecurity groups represent the common characteristics to all entities of Mobile IP. The mipSystem group shows the UP/DOWN status and supported packet encapsulation type of each entity, where the mipSecurity group represents the security related information such as security algorithm, security mode, security violation of SPI, etc., exchanged among entities during the registration of Mobile IP. The mipMA group is the common MIB used by 
two types of Mobility Agents (FA and HA), and shows agent specific information such as agent advertisement. The remaining Mobile IP MIB groups (mipMN, mipFA, and mipHA group) provide the entity-specific information.

\section{Autonomic Network Management for Mobile IPv4 Based Wireless Networks}

In this section, we introduce the concept autonomic wireless network management and architecture for Autonomic Wireless Network Management System (AWNMS), which makes SNMP agents to manage wireless networks more intelligently through the self-management functionality.

\subsection{Autonomic Wireless Network Management}

In wireless networks, it is always preferable that mobile nodes are supported the seamless mobility for continuous communication regardless of their handover events. The seamless mobility means that mobile nodes experience the minimized handover latency and can communicate continuously with the communicating partner moving from one network to the new network. For the seamless mobility, network entities in Mobile IPv4 should have active functionalities such as dynamic and adaptive resource management, security negotiation, etc. For example, if the application used in the mobile node requires certain level of QoS for successful communication, network entities should carefully accept the handover mobile node. When the network entity decides that the remaining resource is enough to support the handover mobile node, it can accept the handover request from the previous network entity or the mobile node. Otherwise, the network entity should reject the handover request. Also, if the mobile node initiates the QoS or security negotiation after connecting to the new network, it cannot communicate with its communicating partner until the negotiation procedure is completed. This results in long handover latency.

As the Mobile IPv4 only specifies a basic mobility management scheme in IP networks, several new protocols and schemes have been proposed to overcome the drawback of Mobile IPv4. One of them is the proactive QoS and security negotiations before the actual handover operation. For this, some protocols for information exchange between Mobility Agents or Access Points, such as CARD [9] and InterAccess Point Protocol [14], are proposed. We have integrated these functionalities to the SNMP management framework using the autonomic computing concept [15]. The autonomic management in this paper means that a management system can adaptively monitor, analyze and control its managed systems without any intervention of network managers or operators. In this paper, we address that the functionality of a SNMP agent can be easily extended to have autonomic management functionality and thus a SNMP management framework can provide required operations for the seamless mobility. In the proposed system, we define this component in the active SNMP agent to support this autonomic management functionality.

The network entities such as Mobility Agents also should have adaptive resource management functionality to efficiently manage their radio resources. When a high priority user requests more resources to the Mobility Agent, the Mobility Agent may examine the radio resource usage of each user and allocate resources to the high priority user by preemptively reducing the allocated resources to the low priority 
users. This resource management functionality is invoked when the user coming into the new network has higher priority than the existing users and the remaining resource cannot support the user's request. This operation also can be integrated to the SNMP management framework using the autonomic network management concept; the selfmanagement in the proposed system. In the proposed system, the pre-defined thresholds and the radio resource usages are stored and retrieved to/from the integrated information repository. The active SNMP agent periodically monitors and updates this information based on the basic SNMP operation. If the active SNMP agent exchanges this information with neighbor agents, it may recommend the mobile node to handover to the neighbor network in order to distribute the network load.

In summary, the autonomic concept of the active SNMP agent has two components; the self-management and the manager function which is the ability to exchange information with neighbor agents.

\subsection{Architecture of the Autonomic Wireless Network Management System}

Fig. 3 illustrates the architecture of AWNMS, where SNMP agents have the manager functionality to autonomously manage target systems and networks through a selfmanagement scheme. The self-management means that a SNMP agent, which is referred to as an 'active SNMP agent' in this paper, can dynamically configure managed systems without the manager or the network operator.

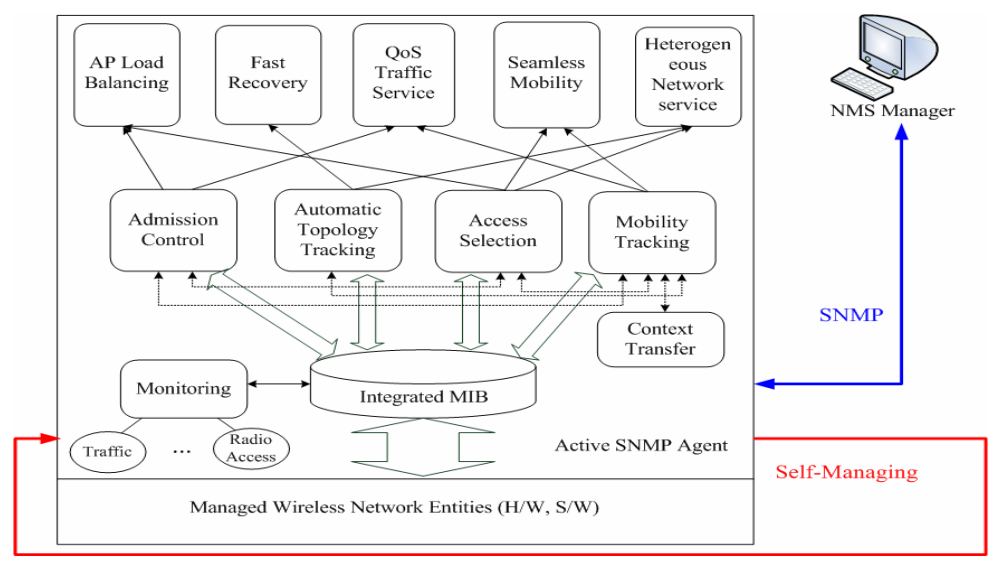

Fig. 3. The architecture of AWNMS

The Integrated MIB (shown in Fig. 3) is a logical management information repository which is maintained by an active SNMP agent through gathering and combining individual information of pre-defined MIBs such as Mobile IP MIB and MIB-II. Therefore, the Integrated MIB operates as the meta-MIB and the basis of the other components. The managed objects of the Integrated MIB, whose example instance is illustrated in Table 2, provide more useful and intuitive information to users than the basic MIBs. As shown in Table 2, when the manager needs the network load information, it just retrieves the information of FALoad managed object through the active SNMP agent. But, without this integrated MIB, we may have to make the 
Table 2. An example instance of managed objects of an Integrated MIB

\begin{tabular}{|c|c|c|}
\hline $\begin{array}{c}\text { Management } \\
\text { Object }\end{array}$ & Information Sources & Description \\
\hline MNLocation & $\begin{array}{c}\text { mnFATable and mnRegistrationTable in } \\
\text { Mobile IP MIB }\end{array}$ & $\begin{array}{c}\text { Movement history of each MN. It is maintained } \\
\text { as table structure. }\end{array}$ \\
\hline FALoad & $\begin{array}{c}\text { faVisitorTable in Mobile IP MIB and } \\
\text { ifTable in MIB-II }\end{array}$ & $\begin{array}{c}\text { Network load information in each FA. It counts } \\
\text { the number of bytes destined to each mobile node. }\end{array}$ \\
\hline NeighborAgent & $\begin{array}{c}\text { ipRouteTable in MIB-II and newly } \\
\text { defined topology information }\end{array}$ & $\begin{array}{c}\text { Neighbor mobility agents information of each } \\
\text { mobility agent. }\end{array}$ \\
\hline TunneledTraffic & $\begin{array}{c}\text { haMobilityBindingTable in Mobile IP } \\
\text { MIB and ifTable in MIB-II }\end{array}$ & $\begin{array}{c}\text { Number of transmitted bytes to each MN using } \\
\text { tunneling. }\end{array}$ \\
\hline
\end{tabular}

manager to gather separately each information and process them, and this makes implementing the SNMP manger even harder and more complex.

In wireless networks, information exchange such as context transfer between network elements is required for efficient mobility management [9, 10]. As the manager can exchange information with other managers, the manager function of the active SNMP agent also can exchange mobility related information, such as context and security information, with neighbor agents having the manager function (Context Transfer component in Fig. 3). The Context Transfer component also can exchange the network capability information with each other.

Based on the Integrated MIB, the active SNMP agent provides four basic components as shown in Fig. 3; admission control, automatic topology tracking, access selection, and mobility tracking. Each component provides useful information to the manager function of the active SNMP agent. As a general admission control function for wireless networks, the Admission Control component provides the network status information such as currently used radio resources and required resources to support new communications. The Integrated MIB can have the resource utilization information using the monitoring component. The Automatic Topology Tracking component provides the network connectivity information. When we consider the node mobility in the Mobile IPv4 network, the network topology discovery functionality becomes more important in the wireless network management system. This information can be retrieved through the general MIB (IP group in MIBII) and Mobile IP MIB (MN/HA/FA registration group in Mobile IP MIB). The Access Selection component provides the network capability information of neighbor networks. This component helps the network discovery and evaluation of the target network to which the mobile node will handover operations required by the handover operation and provides base information to determine the handover is triggered by network-based decision or by node based decision. Finally, the Mobility Tracking component provides the mobility history and pattern information of mobile nodes. The mobility history information is stored in the Integrated MIB through comparing the care-of address information of the mobile node in the Mobile IP MIB. Using the mobility pattern of each mobile node, the active SNMP agent can exchange the QoS and security information prior to the actual handover for fast negotiation and reduction of the handover latency. 
The manager function of the active SNMP agent provides five basic functions based on above components as shown in Fig. 3; AP load balancing, fast recovery, QoS traffic service, seamless mobility, and heterogeneous network service. The AP load balancing and QoS traffic service focus on the communication quality of mobile nodes, and fast recovery, seamless mobility and heterogeneous network service focus on the node mobility. In wireless networks managed by the AWNMS, each AP can maintain its own measurement information such as the number of servicing mobile nodes, maximum capacities and the measure of load, and the neighbor APs exchange the maintained information with each other. Based on this collected information, the AWNMS conducts AP load balancing and QoS traffic service. In AWNMS, the active SNMP agents on APs regulate the load of each AP using the admission control and access control components. The active SNMP agents also maintain user QoS profile and application QoS profile in the Integrated MIB, and adjust QoS parameters or decide to switch network access for better QoS using the mobility tracking and admission control components.

In the Mobile IPv4 based wireless networks, the node mobility should be supported and transparent to users. In AWNMS, the active SNMP agent utilizes the node's mobility pattern to support a seamless, transparent handover using the mobility tracking component. The active SNMP agent can predict the node movement based on the mobility pattern, and makes the environments to communicate immediately through the negotiation with node's profile and QoS profile. The negotiation of node profiles can be conducted between different radio access networks based on the automatic topology tracking and access selection components. Therefore, the AWNMS can support the heterogeneous network service and fast recovery as well as the seamless mobility.

\section{Implementation}

In this section, we present the Mobile IP MIB and Integrated MIB implementation, and the implementation details of the prototype AWNMS system. Before the development of the proposed wireless network management system, we have developed Mobile IPv4 system which consists of HA and FA modules on Linux and MN module on Linux and Windows [10]. We extended the Mobile IPv4 modules to support the Mobile IP MIB completely and some advanced functions such as the tunneled information. Each MN has IEEE 802.11b interface and associated with ORiNOCO AP 2000 product. We used AdventNet SNMP library [11] for SNMP functions and JRobin RRDtool [12] for web-based GUI.

The prototype system gathers information from the common entity information to the entity specific information based on the Mobile IP MIB. However, there are some limitations to just gather the Mobile IP MIB for efficiently managing the node mobility. For example, a mobility management system needs the information on where/when/how much service the mobile node receives during handovers with Mobile IPv4 because this information can be used to track the node mobility. However, network management system has the deficiency for evaluating the amount of serviced data packets through entities of Mobile IPv4, since there is no defined MIB to retrieve that information and already defined received data information in MIB-II includes all received data packets regardless of Mobile IPv4. Thus, we added 
the tunneled Mobile IPv4 information in the existing interface information MIB (e.g., MIB-II interface group) to classify the amount of serviced traffic from entities of Mobile IPv4. Then we developed the mobile node's mobility tracking function based on tunneled traffic information of mobile nodes and binding cache information of mobility agents.

\subsection{Functionalities of the Prototype System}

When we implemented the prototype system, we focused on functions related to the node mobility in the AWNMS and the Mobile IPv4. Thus, the prototype system supports the following functionalities; the network topology auto-discovery, binding cache and visitor list management, mobility tracking, and tunneled packet load information function.

- Network Topology Auto-Discovery Function: The SNMP-based network topology discovery scheme has been implemented in the "Argus" project [13]. When the network management system needs a topology information, it first gets and analyzes the MIB information of the default router. Based on the MIB analysis, the manager can know node and router information in the network connected to the default router. This operation continues to newly found routers and finally the manager can know the subnet and node information to which subnet it belongs.

- Binding Cache and Visitor List Management Function: In the Mobile IPv4, the HA and FA manage information about mobile nodes called the binding cache and visitor list. The binding cache and visitor list management is important to support a node mobility and traffic tunneling in the Mobile IPv4. The prototype system provides management function to access, update, and delete information related to binding cache and visitor list through the SNMP GET/SET methods.

- Mobility Tracking Function: The prototype system records the history of the node mobility and periodically retrieves the current location information of the mobile node using the Mobile IP MIB. This information is stored into the Integrated MIB and the active SNMP agent creates a mobility history and pattern. Using the mobility pattern of each node, the SNMP agent can provide the recovery of a connection and the fast negotiation of QoS, security and node profiles for reducing the handover latency.

- Tunneled Packet Load Information Function: The tunneling operation in the HA (packet encapsulation) and the FA (packet decapsulation) of the Mobile IPv4 results in processing overhead and affects the system performance. Thus, the tunneled packet load information should be carefully investigated to prevent a malfunction caused by the overloads of Mobility Agents. In the prototype system, the HA and FA gather the tunneled packet size per each mobile node and the network management system shows results to the user through the line plotting chart of transmission rate (bps) as a function of time.

\subsection{Architecture of the Manager}

We have implemented the active SNMP agent based on the conceptual agent architecture shown in Fig. 3. In this section, we describe the details of manager. The manager consists of five modules as illustrated in Fig. 4; Scheduler, Alive-Checker, SNMP-Requester, Event-Handler, and Mobility-Handler. The Alive-Checker module 
periodically checks the availability of managed systems using a PING requestresponse exchange. The SNMP-Requester module periodically sends a SNMP request message to managed systems for gathering the defined MIB information. The interval of these two modules is scheduled by the Scheduler module, which creates a thread on each interval per managed system. The Scheduler module has a thread pool and limits the number of threads executed in parallel. The Event-Handler module is executed when it receives a response of the PING request sent by the Alive-Checker module or the SNMP request message sent by the SNMP-Requester module, and updates the status of the managed system or the corresponding MIB information. The EventHandler module also transfers the updated information to the Mobility-Handler module. The Mobility-Handler module manages the status and the handover event of mobile nodes. Therefore, the Mobility-Handler module checks whether the information is transmitted from the mobile node or not. If the information is about the mobile node, it records the mobility related information such as a location history of the mobile node. The Mobility-Handler also periodically checks whether the mobile node returns to its home or not.

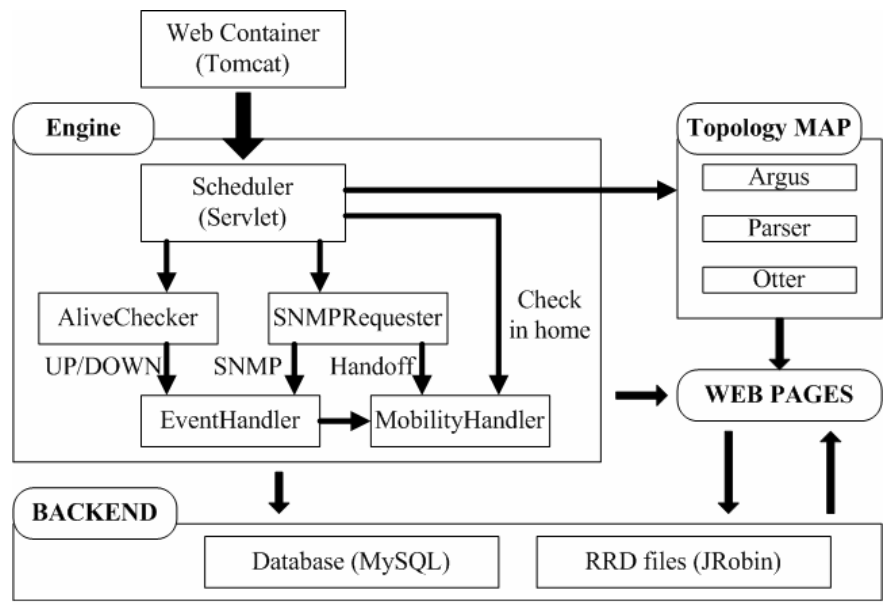

Fig. 4. System architecture of the implemented system

\subsection{Implementation Results}

The main window of the web-based manager is shown in Fig. 5. In the main window, administrators can view the network map, the list of managed systems and the list of important events. When the detailed information of the managed system is needed, administrators can view the information, as shown in Fig. 6, through selecting a specific managed system in the topology map. Fig. 6 shows a node view page of the FA information. In the node view page, administrators can view the basic information of the FA, visitor list information managed by the FA, AP list information in the FA network, and performance charts representing the number of agent advertisement messages which it sent, agent solicitation messages which it received, and agent advertisement messages which it sent responding to the solicitation message from the mobile node. 


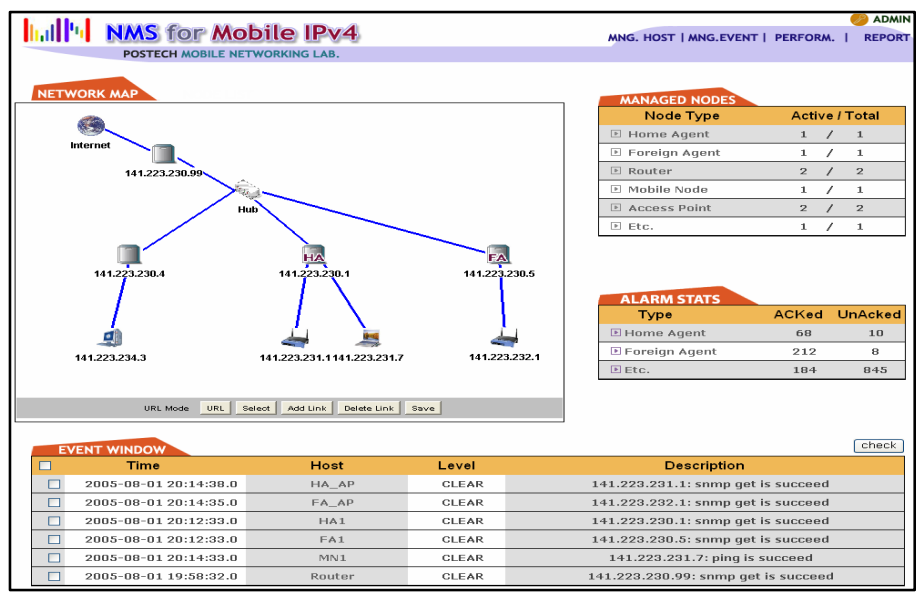

Fig. 5. The main window of the web-based manager

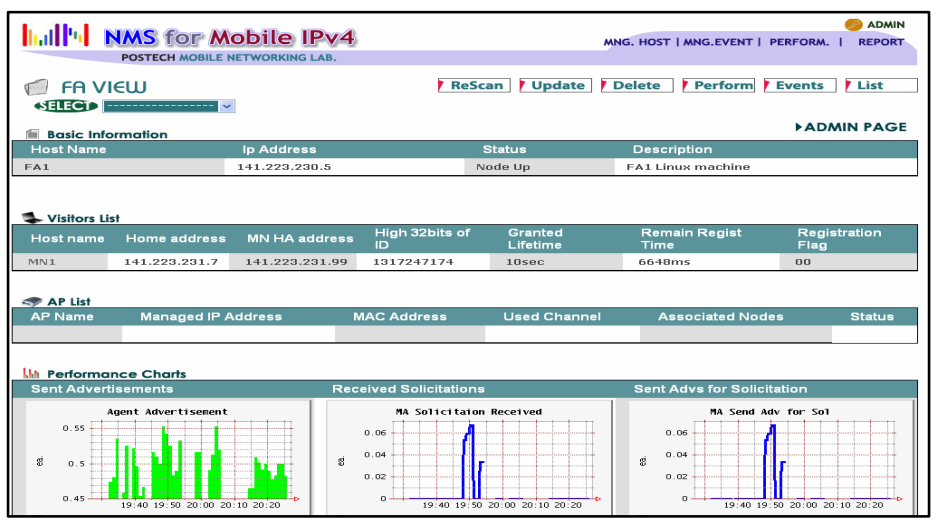

Fig. 6. Node view

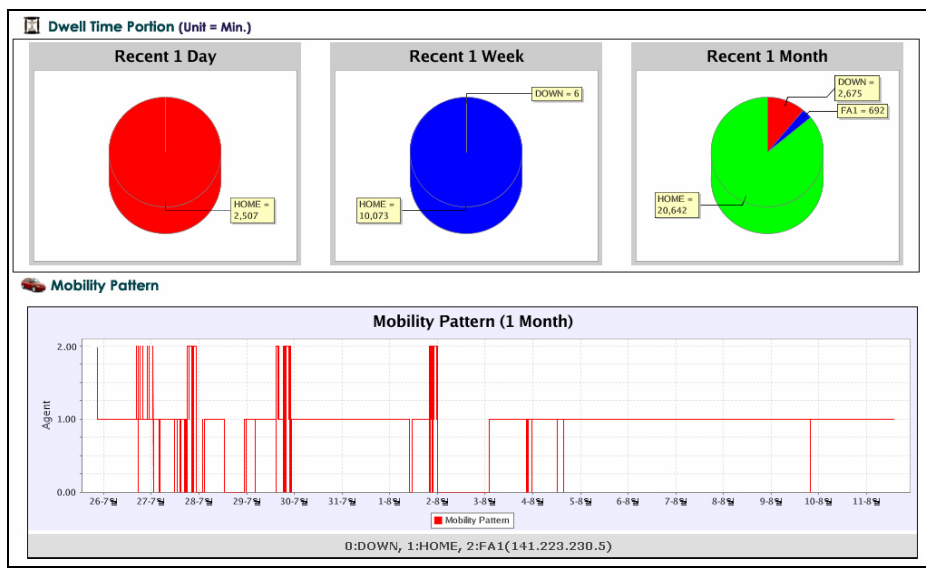

Fig. 7. Mobility tracking view 
Fig. 7 shows a mobility tracking information of a mobile node. In the AWNMS prototype system, the mobility tracking information requires the network connectivity information and location information. Network connectivity of $\mathrm{MN}$ is checked periodically by an Alive-Checker module and location information comes from the gathered MIB information by the SNMP-Requester module. This information is stored into the Integrated MIB, and the AWNMS prototype system can infer whether the mobile node has moved to other networks or not using the information stored in the Integrated MIB. If a mobile node uses a FA address as its CoA, the movement of the mobile node is easily detected by comparing a FA visitor list. If the mobile node is operated in Co-located CoA mode, the management system checks a change of mobile node's address which is dynamically allocated via DHCP server.

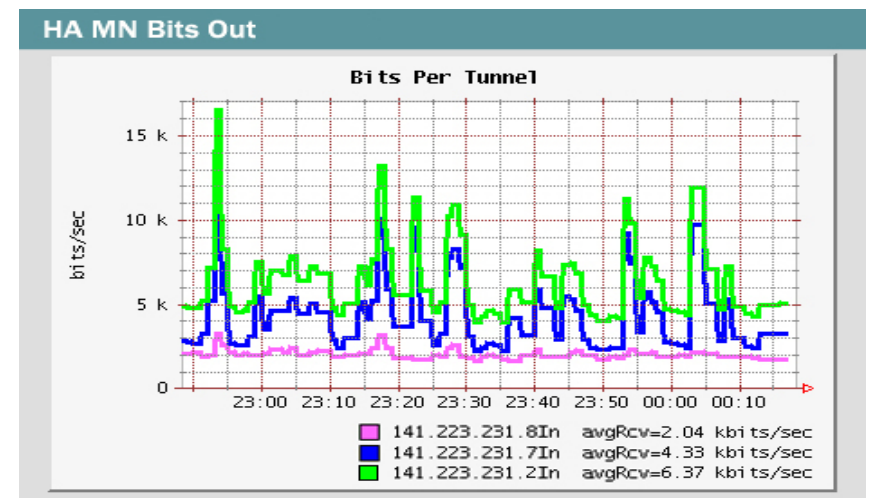

Fig. 8. Tunneled traffic statistics per mobile node in the HA

Although the standard Mobile IP MIB provides the information of each entity of Mobile IPv4, there is no information which enables user to know the exact servicing packets through Mobile IPv4 and the load of Mobile IPv4. Thus, we added the tunneling packet load information to know the exact servicing packets from each agent and the load measurement with Mobile IP MIB. When a tunnel between a HA and FA is created, the new tunnel interface comes into the interface card list. Tunnels are created and destroyed periodically on every handover process although their targets are the same mobile node. In the AWNMS prototype system, tunneled traffic destined to the same mobile node are grouped into the same management entity. Fig. 8 shows the gathering of tunneled information. Using the tunneled forwarding data packets and tunnel interface of each entity, we can create the specific information related to the load information.

\section{Concluding Remarks}

In wireless networks, the Mobile IPv4 based wireless networks are proposed to support a seamless mobility of users. Because there are many dynamic changes and events caused by the node mobility, the active and efficient management system is required to manage wireless networks. However, ordinary network management systems operate in the passive mode. To overcome this passive characteristic, we 
have proposed the Autonomic Wireless Network Management System for the management of Mobile IPv4 based wireless networks. The AWNMS has five components (admission control, automatic topology tracking, access selection, mobility tracking, and Integrated MIB) and provides useful functionalities focusing on the node mobility and the communication quality. The autonomic management concept makes the active SNMP agent adaptively manage its managed systems. In the AWNMS, the active SNMP agent enables the autonomic management concept through two components; the self-management and the manager function which is the ability to exchange information with neighbor agents.

We have also designed and implemented the AWNMS prototype system focusing on the events caused by the node mobility. The prototype system has implemented the Integrated MIB using the Mobile IP MIB and MIB-II, and supports a network topology auto-discovery, binding cache and visitor list management, mobility tracking, and tunneled packet load information functions. We have tested the prototype system in a test-bed network and obtained desirable results. Although the prototype system provides limited functionalities of the AWNMS, our design and implementation can be the basis of developing a full AWNMS. In future, we plan to implement other functionalities of the AWNMS based on the prototype system, and prove the feasibility of the AWNMS in the Mobile IPv4 based wireless networks.

\section{References}

1. IEEE 802.11, Wireless LAN Medium Access Control (MAC) and Physical Layer (PHY) Specifications, Standard, IEEE, Aug. 1999.

2. IEEE 802.16-2004, Air Interface for Fixed Broadband Wireless Access Systems, Standard, IEEE, Oct. 2004.

3. 3GPP Working Group works in progress (Rel6, Rel7).

4. C. Perkins, "IP Mobility Support for IPv4," RFC 3344, IETF, Aug. 2002.

5. D. Johnson et. al., "Mobility Support in IPv6," RFC 3775, IETF, Jun. 2003.

6. J. Case, M. Fedor, M. Schoffstall, and J. Davin (Eds.), “A Simple Network Management Protocol (SNMP)," RFC 1157, IETF, May 1990.

7. William Stallings, "SNMP, SNMPv2, SNMPv3, and RMON1 and 2," $3^{\text {rd }}$ Ed. Addison Wesley, 1999.

8. D. Cong and M. Hamlen, "The Definitions of Managed Objects for IP Mobility Support using SMIv2," RFC 2006, IETF, Oct. 1996.

9. M. Liebsch et. al., "Candidate Access Router Discovery (CARD)," RFC 4066, IETF, Jul. 2005.

10. Dong-Hee Kwon et. al., "Access Router Information Protocol with FMIPv6 for Efficient Handover and Their Implementation," IEEE Globecom 2005, vol. 6, pp. 3814-3819, 2005.

11. AdventNet SNMP Library, http://snmp.adventnet.com/

12. JRobin, http://www.jrobin.org.

13. Project Argus - Network topology discovery, monitoring, history, and visualization, http://www.cs.cornell.edu/boom/1999sp/projects/Network Topology/topology.html

14. IEEE 802.11F, IEEE Trial-Use Recommended Practice for Multi-Vendor Access Point Interoperability via an Inter-Access Point Protocol Across Distribution Systems Supporting IEEE 802.11 Operation, Standard, IEEE, Jul. 2003.

15. M. Mitchell Waldrop, "Autonomic computing: The technology of self-management," IBM white paper, http://www-03.ibm.com/autonomic/library.shtml, 2003. 